\title{
La educación médica en tiempos del covid-19
}

\section{Medical education in times of covid-19}

La pandemia de la covid-19 debido al SARS-COV-2 ha producido una serie de cambios que involucra a toda actividad humana alrededor del planeta. Debido a su alta contagiosidad y propagación, las medidas sanitarias implementadas generan restricciones que afectan el normal desenvolvimiento de estas actividades. Específicamente, el distanciamiento social, es decir mantener una separación de por lo menos de 1-2 metros con respecto a otra persona, ha desencadenado una redefinición de cómo se debe interactuar en un nuevo escenario para el cual no se estaba preparado (1). Sin embargo, aún es incierta la verdadera efectividad de esta medida, estando también pendiente entender los mecanismos de diseminación y el desarrollo de nuevas terapias y vacunas que controlen los efectos del COVID-19 (2). Es un problema de naturaleza global que genera una situación impensada, inédita y que no se ha tenido experiencias similares previas.

Uno de los sectores más afectados es la educación en todos sus niveles, habiéndose suspendido la asistencia a las escuelas, institutos y universidades. Inesperadamente y con muy poca antelación, las personas ya no pueden enseñar o aprender junto a otras personas (3). En ese mismo sentido, la formación médica ha sufrido un impacto significativo generado por diversos factores como la suspensión del acceso a los campus universitarios y a los campos clínicos y socio-sanitarios. La capacidad resolutiva de las instituciones prestadoras de salud (IPRES) se ha modificado dando prioridad a la atención diferenciada en favor de los pacientes con COVID 19 con lo cual hay una disminución significativa de las actividades en áreas de docencia entre las que se incluyen la consulta externa, cirugías electivas, procedimientos diagnósticos y terapéuticos entre otros.

Es preocupante señalar que también existe una considerable disminución del número de docentes por causa de la propia enfermedad o por ser considerado población vulnerable (edad, comorbilidades). Por otro lado, la docencia en servicio se ha visto desplazada por la actividad asistencial y por lo tanto se han suprimido las actividades académicas hospitalarias tales como visitas, rondas, discusiones de casos clínicos, revisiones bibliográficas, entre otros aspectos. Igualmente, se ha perdido la interacción docente-alumno que conllevaba a resolver dudas directamente sobre los hallazgos o evolución de los pacientes o cómo afrontar las preguntas que puedan surgir desde la perspectiva de ellos mismos.

EL distanciamiento social también implica una restricción a la movilización nacional e internacional que afecta los programas de intercambio académico y a las actividades vinculadas a la responsabilidad social universitaria, así como la organización y participación de eventos académicos internacionales entre los que se incluyen congresos, simposios y conferencias.

Los efectos de la pandemia en la educación médica deben ser afrontados sobre la base de una respuesta inmediata, las opciones de ofrecer alternativas a la formación habitual y los posibles cambios y acciones futuras (4). Entonces, frente a esta situación de crisis se plantean dos principales interrogantes: ¿cómo se debe responder en pleno curso de la pandemia? y lo segundo: ¿cómo va a ser la educación médica post pandemia?

\section{La respuesta de las facultades de medicina}

Las entidades formadoras deben comprometerse a buscar la continuación de la formación de los profesionales de la salud apelando a otras modalidades educativas que vele por la seguridad e integridad de los involucrados sin menoscabar la responsabilidad de atender la necesidad de recursos humanos producto de la pandemia.

1 Universidad Peruana Cayetano Heredia. Lima, Perú.

a Decano, Facultades de Medicina, Estomatología y de Enfermería. 
Atendiendo a las inquietudes planteadas, definitivamente este nuevo escenario ha demandado una respuesta urgente por parte de los programas de medicina que han tenido que realizar grandes esfuerzos para llevar a cabo planes de contingencia que minimicen las interrupciones o los profundos cambios que se producen en área de pregrado y residencia y que se han traducido en suspender la participación de los estudiantes en cualquier actividad que involucre pacientes. En esta situación, es que los educadores médicos deben ser creativos en ofrecer alternativas significativas (3).

Comparativamente con otras carreras, tanto los docentes como los alumnos de los programas de ciencias de la salud tienen un rol activo en el desarrollo de las actividades inherentes a la propia disciplina. Es menester replantear el papel de los estudiantes de Medicina no solo en asegurar una formación adecuada sino para adaptarse y convertirse en un agente efectivo que forme parte de los recursos humanos que están enfrentando la crisis sanitaria.

El mayor desafío es tratar de replicar la interacción clínica con el paciente. Esta interacción va desde pasar visita o rondas junto con otros estudiantes supervisados por los profesores hasta la evaluación directa al paciente desarrollando habilidades para establecer una comunicación fluida entre ellos.

Además de los aspectos cognitivos, es necesario asegurar que los estudiantes desarrollen las llamadas competencias blandas como el trabajo en equipo, la empatía, la iniciativa, la compasión entre otros. También deben tener la oportunidad de aprender el manejo racional de recursos limitados que tienen las situaciones de emergencia sanitaria (5).

Esta situación ha dado paso a tener que implementar la virtualización del $100 \%$ de horas de teoría y la mayor cantidad posible de prácticas en laboratorios con el uso de softwares, videos y podcasts tutoriales, simulación clínica virtual, utilizando el contexto covid-19 para la construcción de escenarios. Las sesiones de aula con un número amplio de alumnos han sido reemplazadas por cursos en línea, webinars o actividades en plataformas virtuales con un menor aforo de estudiantes.

Es absolutamente fundamental que todas estas medidas sean de pleno conocimiento de los estudiantes. Que estén perfectamente conscientes del impacto que está teniendo la pandemia en su formación de futuros médicos y como las entidades formadoras y reguladoras del sistema están procurando superar este difícil trance (6).

Una de las principales dificultades en implementar la migración de los planes de estudio al escenario de la virtualidad ha sido la necesidad de contar con una plataforma robusta que dé soporte a un uso masivo de actividades académicas en línea. La mayoría de las universidades cuentan con un recurso tecnológico de esta naturaleza, pero cuyo uso era mayormente incipiente y restringido. Abruptamente se han visto obligados a invertir en herramientas tecnológicas más avanzadas que sirvan de entorno apropiado para dicha virtualización (7).

Por otro lado, el desafío que ha significado para los profesores migrar de la metodología presencial tan arraigada a un nuevo esquema en línea, ha demandado que se instalen programas de capacitación intensiva pero que no resuelven necesariamente la brecha de formación tecnológica y la capacidad de utilizar estas herramientas eficientemente en un período relativamente muy corto. Existe efectivamente lo que se llama "tecnofobia" entre los docentes que les dificulta el uso apropiado de los recursos informáticos (8).

La asimetría del conocimiento digital entre estudiantes y profesores no debe ser un factor más que ahonde la situación de desventaja generacional, sino que se constituya en un facilitador de la labor docente al establecer un trabajo conjunto con los alumnos en el uso eficiente de la virtualidad.

Otro de los problemas es la equidad del acceso al servicio en línea. No todos los estudiantes han tenido la posibilidad de utilizar un dispositivo apropiado como una laptop, tableta o similar ni contar con internet de alta velocidad que les permita acceder en forma conveniente a la oferta académica virtual. Es justo reconocer el esfuerzo de las universidades en tratar de superar esta brecha de conectividad. 
Finalmente, otro reto es bajo que modalidad se debe llevar a cabo las evaluaciones tanto formativas como las sumativas por lo cual se están desarrollando procedimientos evaluativos novedosos en diversas plataformas informáticas que permiten tener un control del examinado para evitar que incurra en una trasgresión de la integridad de la prueba (4).

En el caso particular del programa de medicina de la Facultad Alberto Hurtado, el plan de estudios implica realizar prácticas de diferente índole a lo largo de los siete años que dura la carrera. La proporción teoría/práctica es en términos generales $50 \%$ en los tres primeros años. Para cuarto y quinto año se incrementa la actividad clínica al $75 \%$ y es prácticamente el $100 \%$ en los dos últimos años. Esta situación representa un gran desafío dado que actualmente es casi imposible poder virtualizar todos los componentes prácticos del diseño curricular.

Para los tres primeros años, ha sido posible reemplazar las prácticas de laboratorio con recursos virtuales, sin embargo, es indispensable la presencialidad para los cursos clínicos y la práctica pre-profesional de los alumnos de los años superiores. En ese sentido, se cuenta con unidades de simulación en el campus universitario pero cuyo acceso está parcialmente restringido hasta que el gobierno, a través del Ministerio de Educación, autorice el retorno pleno a las aulas.

Para el caso de las prácticas clínicas y las pre-profesionales, es imperativo poder acceder a establecimientos de salud cuya decisión está en manos de los entes rectores como el Ministerio de Salud (MINSA), EsSalud, las Fuerzas Armadas y Policiales e inclusive las Clínicas Particulares. Recientemente se han dado los dispositivos necesarios permitiendo el retorno progresivo y voluntario de los internos a los campos clínicos para que retomen su formación bajo un esquema muy riguroso de protocolos de bioseguridad (9).

\section{El futuro de la educación médica luego de la pandemia}

Los efectos profundos de la COVID-19 pueden cambiar por siempre la forma en que se educa a los futuros médicos. Se prevé que va a ser muy difícil regresar al mismo escenario de la educación médica antes de la pandemia, básicamente porque se habrá conocido y utilizado más los recursos tecnológicos para impartir la enseñanza en medicina y muchos de ellos reemplazarán definitivamente algunos de los métodos tradicionales (10).

La transformación estará en función de cuál va a ser el nuevo comportamiento de los individuos y de los sistemas sociales; también van de la mano con el tiempo que se va a necesitar para tener bajo control a la pandemia. Actualmente, la incertidumbre es el principal escollo que impide definir qué cambios se van a producir y permanecer en lo sucesivo.

¿Qué hace que ocurra la transformación? las nuevas formas de enseñanza se hacen rutinarias y se incorporan al trabajo diario de los individuos y los grupos de personas. La clave está en el conocimiento compartido, la importancia y beneficios que los cambios originan y el compromiso de los actores en darle sostenibilidad a las mejoras implementadas. Cada vez hay menos resistencia por estos sistemas y mayor orientación de recursos para poder consolidar estas prácticas (10).

Las entidades formadoras debemos reflexionar sobre cuál es el perfil del médico que queremos, a partir de la pandemia del COVID-19. Previa a esta situación de crisis ya se había planteado la necesidad de poner énfasis en incorporar en el perfil de egreso, algunas competencias y cualidades como procurar dar un entrenamiento integral que involucre la prevención,promoción y recuperación de la salud aunada a una sólida formación científica, ética y moral. Que esté orientado a la vocación de servicio con visos de humanización y profesionalismo en su actuar y que sea capaz de desarrollar un aprendizaje autónomo y permanente siendo gestor de su propia información (11). 
A estas competencias se les debe añadir ahora la habilidad de afrontar los retos que el sistema sanitario exige actualmente bajo el concepto amplio de la Salud Públicay la necesidad de reorientar y consolidar la atención primaria en salud. Ser capaz de diseñar y mejorar continuamente el cuidado de la salud incorporando el manejo eficiente de la información y alta tecnología a la vez que se busca eliminar las disparidades y discriminación al proveer una atención de calidad (12).

En el futuro, los estudiantes de Medicina de hoy podrán decir que, si bien no estuvieron en la primera línea de acción, progresivamente si fueron parte de la respuesta sanitariaytuvieron a oportunidad de aprender como desempeñarse en un escenario de crisis y desarrollar nuevos niveles de cuidado y atención acorde con las nuevas demandas emergentes de salud (6).

La pandemia también ha revelado un hecho inusitado en relación con la predisposición de todas las escuelas de Medicina a compartir sus experiencias vividas al afrontar los estragos que ha producido el COVID-19, que soluciones han podido ir implementando y cuales han sido sus resultados. Debe buscarse que esta actitud colaborativa sea sostenible en el tiempo y eventualmente convertirse en una tendencia permanente en forma de una contribución global al mejoramiento de la oferta académica que se ofrece actualmente.

\section{Reflexiones finales}

La pandemia de la COVID-19 ha producido un impacto significativo en todos los sectores a nivel global que incluye a la educación y a la salud.

La educación médica ha sido uno de los sistemas que ha sufrido una mayor disrupción al haberse establecido el distanciamiento social como una de las medidas sanitarias que afecta continuar con la formación de los estudiantes según el esquema que tradicionalmente se había venido utilizando.

Es absolutamente necesario que se produzca una reconfiguración de los estudios en Medicina que incorpore nuevas alternativas de formación basados en la tecnología disponible como es la educación en línea y la virtualización de los contenidos.

Los programas de Medicina están haciendo esfuerzos significativos para implementar estas alternativas tecnológicas no sin encontrar obstáculos como la poca disposición de un grupo de docentes a migrar a este nuevo escenario, la posibilidad contar con una plataforma que dé un soporte apropiado y tener una facilidad de conectividad que permita una cobertura de acceso equiparable a todos los miembros de la comunidad universitaria.

Es indudable que luego de la pandemia, la educación médica debe sufrir una gran transformación adoptando nuevas alternativas tecnológicas que permitirán desarrollar nuevos modelos de formación que busquen adquirir competencias clínicas específicas en los estudiantes en lugar de los métodos tradicionales que se basan en el aprendizaje producto de un mayor tiempo de exposición a los escenarios clínicos.

Si bien previamente existía cierta timidez o reparo para implementar la virtualidad como alternativa de formación de los estudiantes, la crisis actual debe convertirse en una excelente oportunidad en darle un nuevo cariz a la educación médica.

Manuel Rodríguez Castro ${ }^{1, a}$ 


\section{REFERENCIAS BIBLIOGRÁFICAS}

1. Eva KW. Strange days. Med Educ. 2020;54(6):4923.

2. Bauchner H, Sharfstein J. A Bold Response to the COVID-19 Pandemic: Medical Students, National Service, and Public Health. JAMA. 2020; 323(18):1790-1.

3. Costa MJ, Carvalho-filho M. A new age for medical education after COVID-19. FEM. 2020; 23(2):55-7.

4. DocTutor. Oportunidades que ofrece la Pandemia en la Educación Médica. 2020. p. 17-9.

5. Asociación Mexicana de Facultades y Escuelas de Medicina. El papel del estudiante de medicina ante la pandemia por COVID 19. Una responsabilidad compartida [Internet]. 2020. Disponible en: www. amfem.edu.mx

6. Herrera P, Toro C. View of Medical education during the COVID -19 pandemic global initiatives for undergraduate, internship, and medical residency. pdf. Acta Méd Peru. 2020; 37(2):169-75.

7. Rajab MH, Gazal AM, Alkattan K. Challenges to Online Medical Education During the COVID-19 Pandemic. Cureus. 2020;12(7):e8966. doi:10.7759/ cureus. 8966.

8. Samaraee A Al, Samaraee A Al. The impact of the COVID-19 pandemic on medical education. Br J Hosp Med. 2020; 81(7):1-4. doi: 10.12968/ hmed.2020.0191.

9. El Peruano. Decretos de urgencia. Normas Leg [Internet]. 2020;(15):358281-2. Disponible en: https://busquedas.elperuano.pe/download/ url/decreto-de-urgencia-que-establece-medidasexcepcionales-y-te-decreto-de-urgencia-n090-2020-1874820-3

10. Goh PS, Sandars J. A vision of the use of technology in medical education after the COVID-19 pandemic. Med Ed Publish. 2020; 9(1):1-8.

11. Risco de Dominguez G. Educación Médica: Nuevas Tendencias, Desafíos Y Oportunidades. Rev Digit Invest Docencia Univ. 2007; 3(1):20. DOI: https:// doi.org/10.19083/ridu.3.20

12. Lucey C, Johnston C. The Transformational Effects of COVID-19 on Medical Education. JAMA. 2020; 324(11):9-10. 